(C) 1993 IEEE. Personal use of this material is permitted. However, permission to reprint/republish this material for advertising or promotional purposes or for creating new collective works for resale or redistribution to servers or lists, or to reuse any copyrighted component of this work in other works must be obtained from the IEEE.

\title{
Flat Beam Studies in the SLC Linac*
}

\author{
C. Adolphsen, F.-J. Decker, J. T. Seeman \\ Stanford Linear Accelerator Center \\ Stanford University, Stanford, California 94309 USA
}

\begin{abstract}
The Stanford Linear Collider (SLC) was recently converted to flat beam operation $\left(\gamma \varepsilon_{\mathbf{x}}=10 \gamma \varepsilon_{\mathbf{y}}\right)$, producing a factor of two increase in luminosity 1 . In this paper we review the results of flat beam studies in the SLC Linac. In summary, the injected beams from the damping rings had invariant horizontal emittances as low as $\mathbf{3 0} \mathrm{mm}$-mrad and invariant vertical emittances as low as $2 \mathrm{~mm}$-mrad. The emittances measured at the end of the linac after tuning for $3 x$ $10^{10}$ particles are about 5 to $8 \mathrm{~mm}$-mrad vertically and 40 to $50 \mathrm{~mm}$-mrad horizontally. Flat beam operation began 3/17/93.
\end{abstract}

\section{Introduction}

Two bunches $\left(\mathrm{e}^{-}\right.$and $\left.\mathrm{e}^{+}\right)$are accelerated in the linac from 1.19 to $47 \mathrm{GeV}$ in $3 \mathrm{~km}$ of $S$-band RF structure. Special measurements, component tolerances, and controls are needed to maintain the initial 10 to 1 emittance ratios. Transverse wakefield damping (BNS) is used in the first two-thirds of the linac to control wakefield growth from beam injection jitter ${ }^{2}$. A small energy spread $(0.26 \%)$ at $47 \mathrm{GeV}$ is made by careful overall klystron phasing ( $+/-0.5$ degrees). Along the linac, eight feedback loops $\left(x, x^{\prime}, y, y^{\prime}\right.$ for two beams) updating at 30 $\mathrm{Hz}$ maintain the trajectory to about $25 \mu \mathrm{m}$. Emittance measurements are made at three locations along the linac (at $1.2,15$, and $47 \mathrm{GeV}$ ) using three sets of four wires scanners, each with an emittance resolution of $0.5-0.75 \mathrm{~mm}$-mrad. Typical vertical beam sizes $\left(\sigma_{\mathrm{y}}\right)$ at $47 \mathrm{GeV}$ are 35 to $60 \mu \mathrm{m}$. The emittance and TWISS parameters are measured automatically by the control computer in all parts of the SLC about every 30 minutes and recorded in long term history buffers for analysis. The linac emittance histories showing the start of flat beam running on 3/17/93 can be seen in Figure 1.

\section{Emittance Optimization}

The study of flat and round beam cases allows the comparison of various types of linac emittance enlargements. Betatron mismatches with filamentation cause multiplicative enlargements: $B_{\operatorname{mag}}=\varepsilon / \varepsilon_{0}$. Dispersion, wakefields, and coupling, followed by filamentation, generates additive growth: $\varepsilon=\varepsilon_{0}+f / 2$. Sqrt(f) is the normalized 'kick' amplitude.

Prior to the conversion to flat beams, the emittance enlargement effects along the linac were feared to be mostly additive. Thus, changing to flat emittances would lead to minimal overall luminosity gain. However, as it turned out, the multiplicative effects dominated the residual emittance increases with round beams and significant emittance reductions were made with the new smaller initial emittances.

The dispersion and beta-functions are carefully tuned in the ring-to-linac transport line to provide the best injected beam emittances ${ }^{3}$. The linac klystron phases and accelerating gradient must be accurately maintained (a few degrees and 5\%, respectively) in the first few hundred meters to avoid chromatic emittance enlargement. Furthermore, the strengths of three quadrupoles in the early linac were empirically adjusted by up to $10 \%$ to minimize the emittances at the $15 \mathrm{GeV}$ location. The linac support girders were mechanically clamped to reduce component vibration ${ }^{4}$ below $100 \mathrm{~nm}$. These clamps were set at full tunnel temperature to avoid systematic transverse component of fsets leading to dispersion and wakefield errors.

The emittance growth from residual dispersion and wakefield effects is reduced by cancellation using "trajectory bumps" located along the linac ${ }^{5}$. In the tuning process, the SLC operators try to minimize the beam emittance at two downstream wire scanner locations ( $15 \mathrm{GeV}$ and $47 \mathrm{GeV}$ ). The setpoints of the linac feedback loops are adjusted for this purpose. For each loop, there is a position and angle setpoint, which when varied, generates a betatron oscillation that extends downstream until the next feedback loop removes it. First, the setpoints of the loop at $8 \mathrm{GeV}$ are tuned looking at emittance and beam tail changes at the $15 \mathrm{GeV}$ point. Second, the setpoints at $16 \mathrm{GeV}$ are used to reduce the emittance at 47 GeV. Occasionally, other loops are tried. Typically, 8 to 12 distinct "trajectory bumps" are in use at any given time with amplitudes of order 100-200 $\mu \mathrm{m}$ with lengths of 200 to 800 $\mathrm{m}$. Most of these bumps are stable to $90 \%$ in amplitude over several weeks; however, fine tuning $(10 \%)$ is often done to track hour-by-hour and diurnal changes. For vertical emittances of order $6 \mathrm{~mm}$-mrad, bump control at the $25 \mu \mathrm{m}$ level is needed. The tuning procedure typically requires $15-30$ minutes per beam. Measured data from tuning two representative feedback setpoints at the $16 \mathrm{GeV}$ location to reduce $\gamma E$ at 47 $\mathrm{GeV}$ are shown in Figure 2. Note that the increase in the vertical emittance with bump amplitude is about the same as that of the horizontal because the wakefield and dispersion induced growth is nearly additive for small induced errors.

\section{Emittance versus Beam Charge}

In the fall of 1992 the SLC was operated at $10 \mathrm{~Hz}$ to study the emittance increase along the linac versus beam charge. At $10 \mathrm{~Hz}$ a ring store time longer than the nominal $(1 / 120 \mathrm{sec})$ is possible, decoupling the exit emittances from the entrance emittances, so smaller values are achieved. The electron results with a mixture of short and long store data are shown in Figure 3. (Positron data is similar.) The increase in the emittances at the entrance to the linac with current is due to bunch lengthening in the damping ring (which doubles $\sigma_{z}$ and makes $\sigma_{\mathrm{E}} / \mathrm{E} 50 \%$ higher) and chromatic effects in the bunch compressors. An increase in emittance along the linac was observed, although there was not sufficient beam time to perform all the tuning techniques described above. Thus, these curves may over estimate the possible emittances. One example is from Figure 1 where an incoming vertical invariant emittance of order $3.5 \mathrm{~mm}$-mrad can be maintained over long

* Work supported by the Department of Energy contract DE-AC03-76SF00515. 

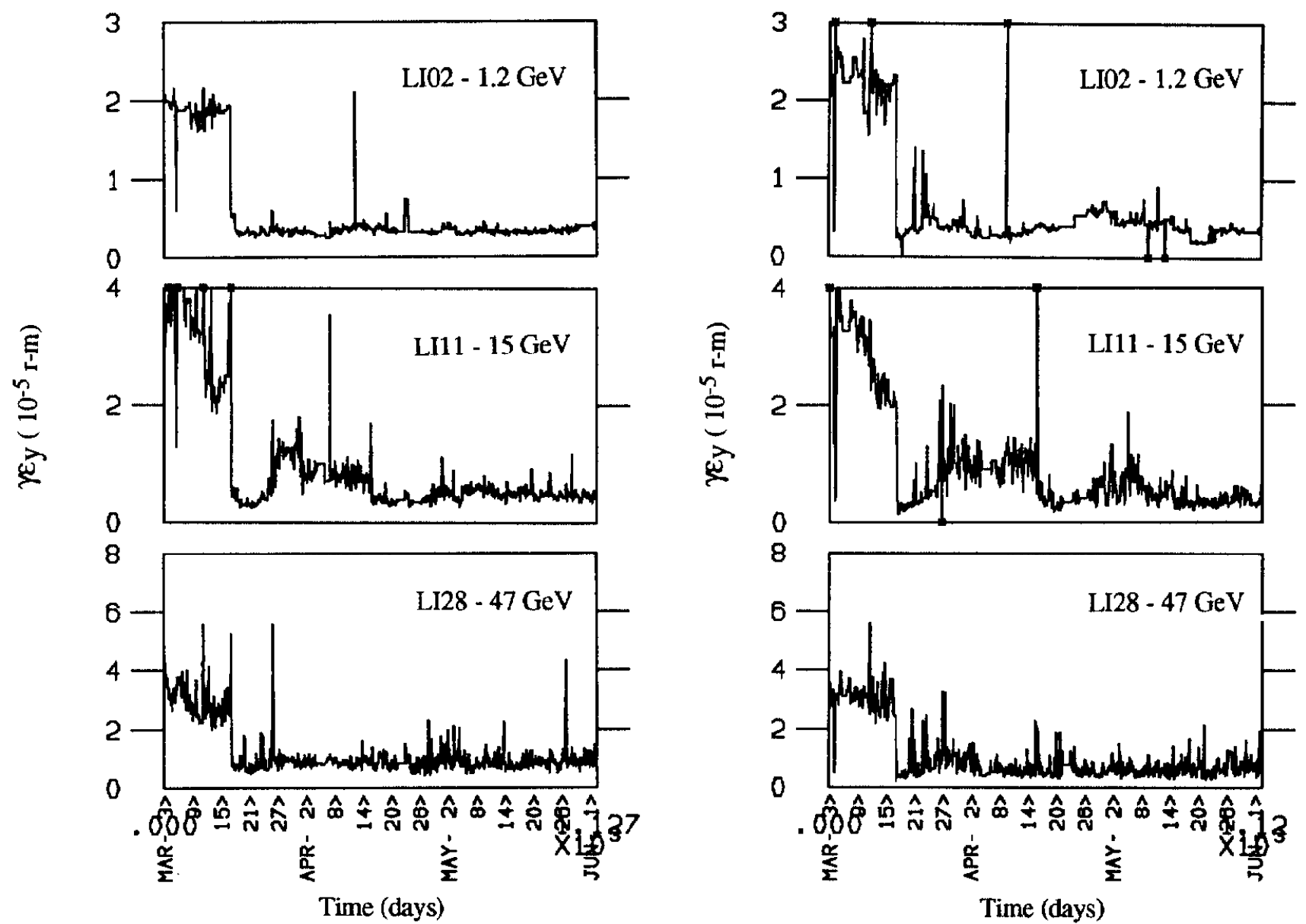

Figure 1 Histories of SLC invariant vertical emittances for $\mathrm{e}^{-}$(left) and $\mathrm{e}^{+}$(right) at $3 \times 10^{10}$ at linac locations: entrance (1.2 GeV, top), $1 \mathrm{~km}$ (15 GeV, center), and exit at $3 \mathrm{~km}$ (47 GeV, bottom). The period is March 3 - June 2, 1993.
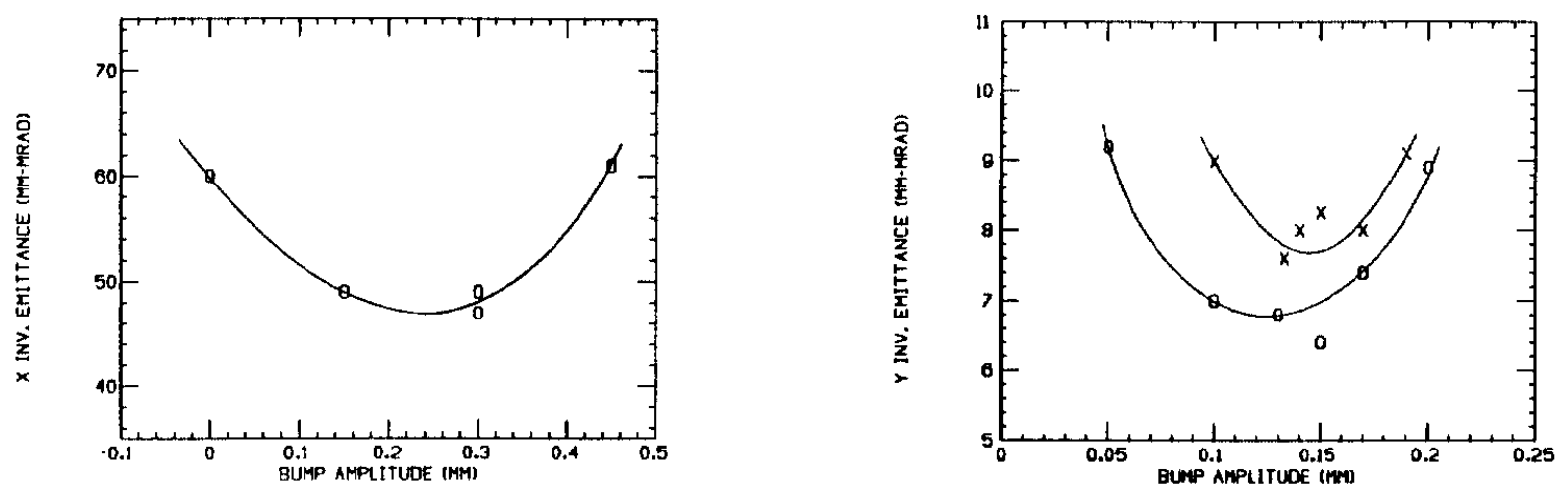

Figure 2 Measured horizontal and vertical emittances for electrons at $47 \mathrm{GeV}$ as a function of amplitude of respective oscillations induced from $1000 \mathrm{~m}$ to $1800 \mathrm{~m}$. Two independent vertical data sets are shown.

times during collisions at $3 \times 10^{10}$ particles per bunch with only 50 to $100 \%$ enlargement along the linac. The incoming horizontal emittance can be maintained at about 35 . mm-mrad with just $5-15 \mathrm{~mm}-\mathrm{mrad}$ increase along the linac. Finally it should be noted that vertical emittances of about $2.0 \mathrm{~mm}$-mrad have been produced at $47 \mathrm{GeV}$ at $1 \times 10^{10 / \text { bunch. }}$

\section{Simulations}

From previous measurements and simulations, we believe that the wakefields generated from misaligned accelerator structures to be the dominate growth mechanism at high currents. In the simulation model, we assume that the accelerator sections are misaligned independently at each end with $300 \mu \mathrm{m} \mathrm{rms}$ offsets. The position monitors and quadrupoles are assumed to have $100 \mu \mathrm{m}$ ms errors.

With these misalignments, simulations of the beam transport were done to compute their effect on the beam emittance ${ }^{6}$. In addition, beam steering and tuning effects were included. As in the SLC, a one-to-one steering procedure was used, where a dipole near each focusing quadrupole is adjusted to zero the BPM reading at the next focusing quadrupole. 

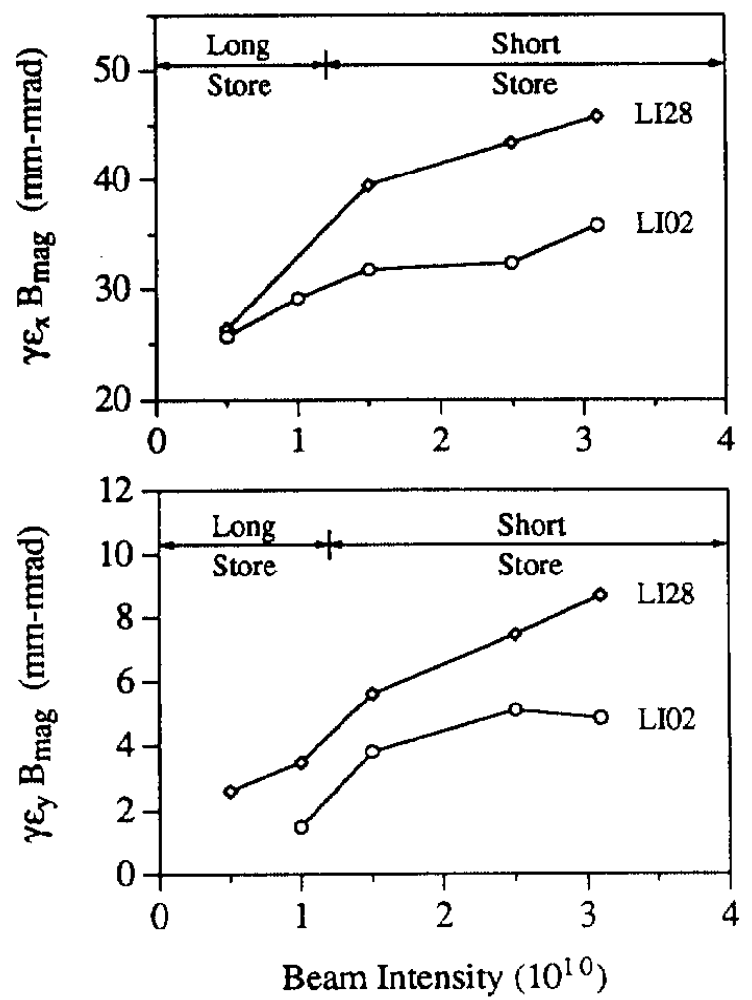

Figure 3 Measured horizontal (upper) and vertical (lower) emittances as a function of beam charge at $47 \mathrm{GeV}$.

Trajectory bump tuning, as described above, was also done first at $15 \mathrm{GeV}$ and then at $47 \mathrm{GeV}$, but a least-squares procedure is used to fit for the effective feedback setpoint changes corresponding to the minimum emittances. A beam with a vertical invariant emittance of $3.5 \mathrm{~mm}$-mrad and an intensity of $3.0 \times 10^{10}$ was assumed to be injected on-axis into the linac.

The simulation program was used to compute the fractional vertical emittance enlargements at $47 \mathrm{GeV}$ for many misalignment configurations. Figure 4 shows the resulting distributions for three linac conditions: 1) no BNS damping and no bumps, 2) BNS damping with no bumps, and 3) BNS with bumps. Thus, BNS damping helps emittance enlargement by a factor of about two and the further addition of trajectory bumps helps by an additional factor of six, a large gain! The mean of the final distribution corresponds to a $52 \%$ growth, comparable to the vertical results of the SLC as in Figure 1. [There is slightly poorer agreement when comparing the horizontal data and simulation where a measured growth of $20 \%$ or more at $15 \mathrm{GeV}$ was measured when less than $10 \%$ was expected. The source of this growth is under investigation but may simply be insufficient tuning.] A final note: if the linac were operated without the large energy spread from the BNS phase configuration, the tuning would further reduce the emittance growth (on average, about $25 \%$ at $15 \mathrm{GeV}$ and $60 \%$ at $47 \mathrm{GeV}$ ). This is the opposite to what happens with one-toone steering only, where zeroing the BNS phases leads to a $30 \%(200 \%)$ larger growth at $15 \mathrm{GeV}(47 \mathrm{GeV})$. This difference may be related to the nearly 'auto-phased' beam in the upstream end of the linac where the tail growth generated from betatron oscillations is nearly suppressed, making the tuning less efficient.
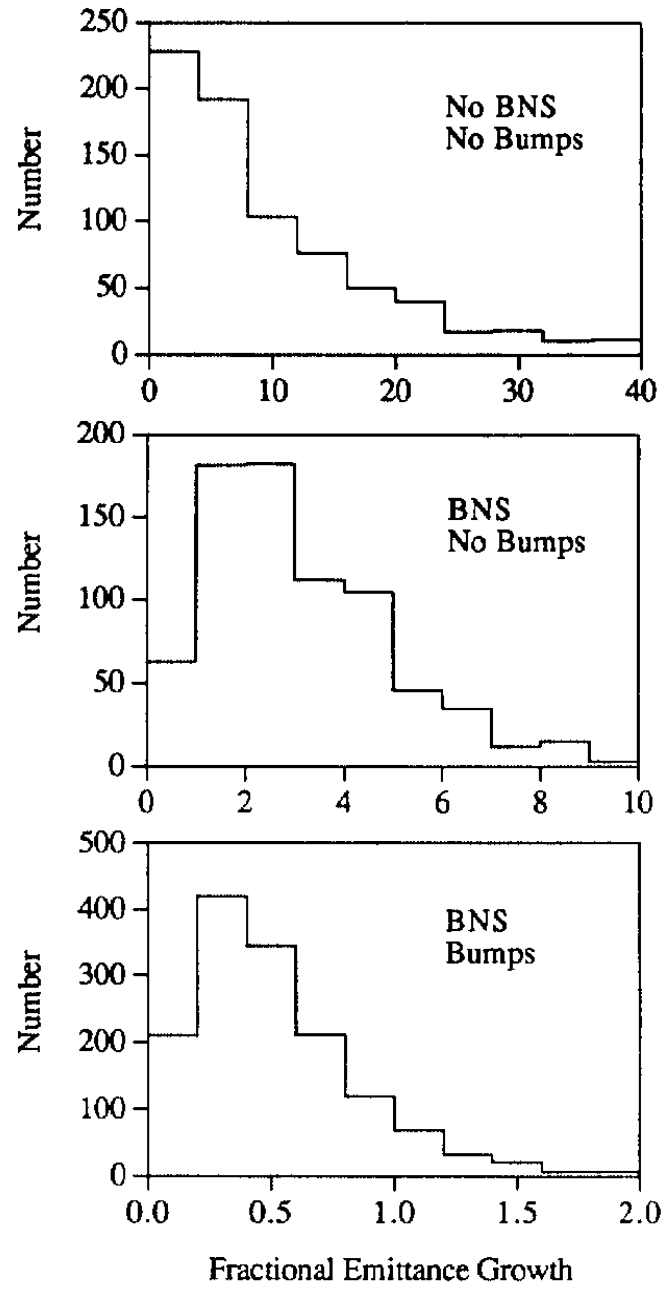

Figure 4 Distributions of final enlargement factors for vertical emittance for simulated linacs with errors without BNS damping (top), with BNS damping (center), and with BNS damping and linac bumps at two locations (bottom).

\section{References}

1) C. Adolphsen, et al., Flat Beams in the SLC, IEEE PAC 93, Washington, DC 1993.

2) J. Seeman, et al, Measured Optimum BNS Damping Configuration of the SLC Linac, Proceedings of 1993 Part. Accel. Conf., Washington, D.C., 1993.

3) F.-J. Decker, et al., Dispersion and Betatron Matching into the Linac, IEEE PAC 91, San Francisco, May 1991, p. 905 .

4) J. Seeman, R. Holtzapple, and M. Ross, Induced Beams Oscillations from Quadrupole Vibrations in the SLC, Proceedings of 1993 Part. Accel. Conf., Washington, D.C., 1993.

5) J. Seeman, F.-J. Decker, and I. Hsu, The Introduction of Trajectory Oscillations to Reduce Emittance Growth in the SLC Linac, XV Int. Conf. on HE Accel., Hamburg, Germany, 1992, p. 879.

6) C. Adolphsen, K. Bane, and J. Seeman, Effect of Wakefields on First Order Transport in the SLC Linac, IEEE PAC 91 San Francisco, May 1991, p. 3207. 